\title{
A Case of Acute Heart Failure With Intravenous Acetazolamide Administration for Evaluation of Cerebrovascular Reactivity
}

\author{
Hiroyuki Miura ${ }^{a}$,, Shuichi Ono ${ }^{a}$, Koichi Shibutani ${ }^{\text {a }}$, Shinya Kakehata ${ }^{\text {a }}$, Hiroko Seino ${ }^{\text {a }}$, \\ Fumiyasu Tsushima ${ }^{a}$, Akihisa Kakuta ${ }^{a}$, Hiromasa Fujita ${ }^{a}$, Yoshihiro Takai ${ }^{a}$
}

\begin{abstract}
A man in his seventies presented with a history of cerebral infarction with occlusion of the left middle cerebral artery, diabetes mellitus, renal dysfunction, as well as prior myocardial infarction and arteriosclerosis obliterans. About 45 min after intravenous administration of 1,000 $\mathrm{mg}$ of acetazolamide for acetazolamide-augmented cerebrovascular single-photon emission computed tomography, he experienced dyspnea and tightness in his chest which worsened. Hypertension, regular tachycardia, and wheezing and rales were noted. Symptoms and vital signs did not improve despite many lifesaving treatments. In patients with severe coronary artery disease with low ischemic threshold, as in the present case, acetazolamide might reduce peripheral vascular resistance, increase the cardiac output ratio, increase myocardial oxygen consumption by increasing cardiac output, and induce myocardial ischemia with elevation of double product. Although the frequency of acute heart failure due to myocardial ischemia after an acetazolamide challenge may be low, its occurrence in patients with severe arteriosclerotic disease should be routinely considered.
\end{abstract}

Keywords: Acetazolamide; Cerebrovascular reactivity; Acute heart failure; Adverse reaction

\section{Introduction}

Regional cerebrovascular reactivity to acetazolamide (DIAMOX $^{\circledR}$, LEDERLE PHARMACEUTICAL DIVISION, NY, USA, Sanwa Kagaku Kenkyusho CO., LTD., Nagoya,

\footnotetext{
Manuscript accepted for publication May 16, 2013

${ }^{a}$ Department of Radiology, Hirosaki University Graduate School of Medicine, 5 Zaifu-cho, Hirosaki, Aomori 036-8562, Japan

${ }^{\mathrm{b}}$ Corresponding author: Hiroyuki Miura, Department of Radiology, Hirosaki University Graduate School of Medicine, 5 Zaifu-cho, Hirosaki, Aomori 036-8562, Japan.

Email: miuradio@cc.hirosaki-u.ac.jp
}

doi: http://dx.doi.org/10.4021/jmc1315w
Japan) using single-photon emission computed tomography (SPECT) is commonly evaluated in patients with cerebrovascular disease and has proven to be an indispensable diagnostic technique [1-3]. It is generally considered to be simple, easy, and noninvasive examination. Until now there have been a few reports of serious adverse reactions after acetazolamide challenge, and here we report our experience of a case of acute heart failure that occurred after cerebrovascular SPECT with intravenous administration of acetazolamide and required many life-saving treatments.

\section{Case Report}

A man in his seventies without complaints was referred for examination with cerebrovascular SPECT with and without acetazolamide challenge to determine whether revascularization was indicated. He had a history of cerebral infarction about 3 months previously and occlusion of the left middle cerebral artery was noted at that time. He also had diabetes mellitus and renal dysfunction, as well as a prior history of myocardial infarction and arteriosclerosis obliterans.

First, SPECT examination was performed using N-isopropyl-4-iodoamphetamine [ $\left.{ }^{123} \mathrm{I}\right]$ ( ${ }^{123} \mathrm{I}$-IMP) without acetazolamide challenge [4] and no adverse reaction occurred. SPECT examination using ${ }^{123}$ I-IMP with acetazolamide challenge was performed 1 week later in accordance with the Japanese Extracranial-Intracranial Bypass Trial (JET study) [5] using autoradiography method [2, 4, 6]. First, 1,000 mg of acetazolamide dissolved in $20 \mathrm{~mL}$ of normal saline was injected intravenously. Ten minutes after administration, 222 $\mathrm{MBq}$ of ${ }^{123} \mathrm{I}$-IMP was intravenously administered and $10 \mathrm{~min}$ after ${ }^{123}$ I-IMP injection, arterial blood sampling from the right brachial artery was performed. Ten minutes after arterial blood sampling, a SPECT scan was started.

About 45 min after acetazolamide administration, the patient complained of difficulty breathing and tightness in the chest. His condition did not improve and dyspnea became apparent. He was moved to the emergency rescue unit and treatment by cardiologists and emergency rescue doctors was started. First, oxygen inhalation $(10 \mathrm{~L} / \mathrm{min})$ was started. The patient's dyspnea and discomfort did not improve and 


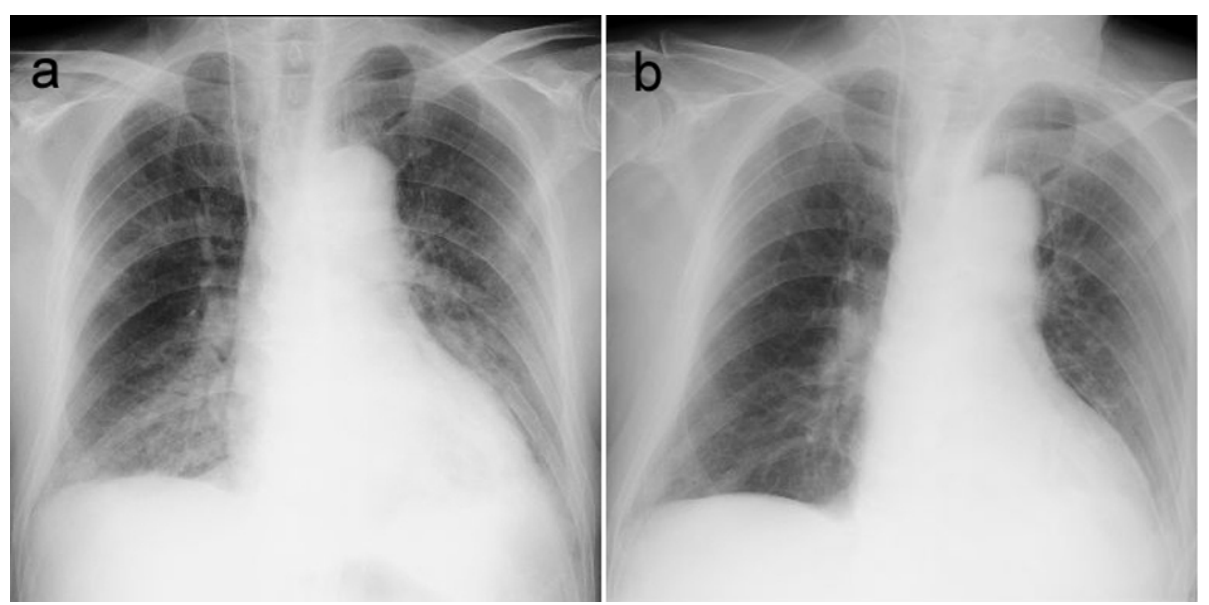

Figure 1. Chest plain radiographs showing a) marked cardiomegaly and increased vascular markings on both lungs on the day of onset, and b) improvement of these findings on day 2.

he gradually became unresponsive to verbal stimuli. Wheezing and rales were heard on pulmonary auscultation. His pulse rate was around 170 beats/min, systolic blood pressure ranged from 200 to $170 \mathrm{mmHg}$, and percutaneous oxygen saturation was around $97 \%$. On electrocardiography, regular tachycardia, narrow QRS, abnormal Q wave on II, III, and $\mathrm{aVf}$, and poor $\mathrm{R}$ wave progression on $\mathrm{V} 1$ - 3 were continuously observed. On ultrasonic cardiography, diffuse left ventricular hypokinetic wall motion was observed and left ventricular ejection fraction was around $27 \%$.

Intravenous administration of $20 \mathrm{mg}$ of adenosine triphosphate twice, administration of seven or 8 direct current shocks, intravenous administration of $0.25 \mathrm{mg}$ of digoxin and $20 \mathrm{mg}$ of furosemide, continuous intravenous infusion of human atrial natriuretic peptide with an injection rate of $0.025 \mu \mathrm{g} / \mathrm{kg} / \mathrm{min}$, again application of a direct current shock, two injections of $2 \mathrm{mg}$ of isosorbide dinitrate, one injection of $1 \mathrm{mg}$ of isosorbide dinitrate, and continuous intravenous infusion of nitroglycerin with an injection rate of $0.3 \mu \mathrm{g} / \mathrm{kg} /$ min were performed one after another. However, his symptoms and signs did not improve quickly.

Over the 3 hour after these emergency treatments, about $700 \mathrm{~mL}$ of urinary output was obtained, and dyspnea improved gradually and systolic blood pressure was reduced to around $100 \mathrm{mmHg}$. His dyspnea disappeared the following day. Although a plain-film chest radiograph taken on the day of onset showed marked cardiomegaly and increased vascular markings of both lungs, these findings improved after admission (Fig. 1).

Transient elevation of serum creatine phosphokinase (CPK) and isoenzyme CPK-MB elevation was observed (Table 1), and given his past history of myocardial infarction, onset of myocardial ischemia was suspected. Although examination of the coronary arteries and cardiac function in detail was recommended, the patient's family did not consent to the procedure. The patient was transferred to another hospital and was lost to follow-up. Therefore the details including the condition of his coronary artery disease could not be confirmed.

\section{Discussion}

Acetazolamide-augmented brain SPECT for evaluation of cerebrovascular reactivity is now a common diagnostic procedure [6]. At the time of this study, mild adverse reactions such as paresthesias, dizziness, and indefinite complaints are sometimes experienced [7]. There have also been a few

Table 1. Change in Serum Creatine Phosphokinase (CPK) and Isoenzyme CPK-MB

\begin{tabular}{|c|c|c|c|c|c|c|}
\hline & Day 0 & 1 & 2 & 3 & 7 & Normal value \\
\hline CPK (U/L) & 264 & 1150 & 1092 & 618 & 56 & $62-287$ \\
\hline CPK-MB (U/L) & 32 & 38 & 13 & 9 & 8 & $0-25$ \\
\hline
\end{tabular}


reports of serious adverse reactions however. A comprehensive literature search identified two reports of enlargement of cerebral infarction $[8,9]$ and one report each of StevensJohnson syndrome [7] and metabolic acidosis [9]. Two cases of adverse reactions involving the circulatory system after acetazolamide challenge have been reported in patients with coronary disease $[10,11]$, but symptoms of those cases were not so severe. In one of these cases, Shimotsu et al performed acetazolamide augmented ${ }^{99 \mathrm{~m}} \mathrm{Tc}-$ hexamethyl-propyleneamine oxime brain and ${ }^{201} \mathrm{TlCl}$ myocardial SPECT simultaneously in a patient with severe coronary artery disease and ${ }^{201} \mathrm{TlCl}$ myocardial imaging with dipyridamole was performed 5 days after acetazolamide stress imaging. A defect seen on acetazolamide stress imaging was seen to be located in the same region as that seen on dipyridamole imaging. In their case, blood pressure and heart rate was unchanged and the patient had no chest pain, ischemic ST-T change, or arrhythmia on electrocardiography during the acetazolamide stress test. However, the patient showed significant ST-T changes on electrocardiography after dipyridamole administration [10]. They subsequently performed the same examination in 9 patients, and showed acetazolamide could cause myocardial ischemia in patients with severe coronary artery disease. In their cases, none of these patients caused myocardial ischemia after acetazolamide administration manifested electrocardiographic ST-T changes, arrhythmia or chest pain [12]. However, the authors could not explain this mechanism [12]. On the other hand, Suzuki et al performed acetazolamide-augmented cerebrovascular SPECT in patients with severe coronary artery disease with continuous intravenous administration of nicorandil, and severe back pain with ST depression on electrocardiography occurred in one case. Although sublingual administration of isosorbide was ineffective, symptoms disappeared $30 \mathrm{~min}$ after intravenous administration of morphine although mild ST depression continued for a further $90 \mathrm{~min}$ [11].

Shimotsu et al speculated that a vasodilation mechanism unrelated to acetazolamide's ability to inhibit carbonic anhydrase is involved in the direct action of acetazolamide on vascular smooth muscle, and that acetazolamide also reduces systolic blood pressure and peripheral vascular resistance and increases the cardiac output ratio in patients with cardiovascular diseases $[12,13]$. They also mentioned that acetazolamide might increase myocardial oxygen consumption by increasing cardiac output, thus inducing myocardial ischemia in patients with severe coronary artery disease with low ischemic thresholds [12]. In contrast, Suzuki et al conjectured that there is little possibility of coronary spasm because of inefficacious sublingual administration of isosorbide, and also mentioned that it seemed unlikely acetazolamide would raise blood pressure and heart late, increase myocardial oxygen consumption, and worsen angina [11].

Although they considered cardiac output would be increased at the onset of angina, this phenomenon was at- tributable to sympathicotonia caused by chest pain, not by acetazolamide, because change in the electrocardiography wave continued even after chest pain disappeared. They considered that acetazolamide affected the tonus of the coronary artery, and that the steal phenomenon occurred not only from dilatation of the coronary artery but also potentialization of nicorandil's pharmacological action [11].

Our patient, however, had no complaint of chest pain, both heart rate and blood pressure were raised, and double product (i.e. systolic blood pressure multiplied by heart rate) of $>30,000$ was continued. Although myocardial ischemia should have occurred in the present case because of elevation of CPK and CPK-MB, the mechanism causing it should be different from that in Shimotsu and Suzuki's cases. It should be considered whether elevation of cardiac output induced myocardial ischemia or whether onset of myocardial ischemia induced acute heart failure. It would seem that there is little possibility that acetazolamide causes myocardial ischemia directly [10-13]. Although the details were unclear, our patient also had systemic arteriosclerosis including severe coronary artery disease. One possible explanation in this case is that acetazolamide might have increased myocardial oxygen consumption by increasing cardiac output, but peripheral vascular resistance might not have been much reduced. This might have caused increased cardiac work, leading to cardiac ischemia because of severe coronary arteriosclerosis with a low ischemic threshold as well as increased myocardial oxygen consumption, followed by acute heart failure with high systolic blood pressure and tachycardia. In addition, renal dysfunction might have aggravated the symptoms as a synergistic effect. There is every possibility that our patient's acute heart failure and myocardial ischemia were affected by acetazolamide administration since he had not only coronary disease but also severe systemic arteriosclerosis.

As a matter of course, preparations should be made for a possible emergency during and after cerebrovascular SPECT with acetazolamide challenge, and patients should be closely observed after the examination. Although the frequency of acute heart failure associated with acetazolamide challenge may be low, the possibility of severe adverse reactions in patients with severe arteriosclerotic disease should be routinely considered.

\section{Funding Statement}

Any company did not support this work.

\section{References}

1. Ito H, Shidahara M, Inoue K, Goto R, Kinomura S, Taki $\mathrm{Y}$, Okada K, et al. Effects of tissue heterogeneity on cere- 
bral vascular response to acetazolamide stress measured by an I-123-IMP autoradiographic method with singlephoton emission computed tomography. Ann Nucl Med. 2005;19(4):251-260.

2. Ohkubo M, Odano I. A comparative study of simple methods to quantify cerebral blood flow with acetazolamide challenge by using iodine-123-IMP SPECT with one-point arterial sampling. Ann Nucl Med. 2000;14(2):115-120.

3. Okudaira Y, Bandoh K, Arai H, Sato K. Evaluation of the acetazolamide test. Vasoreactivity and cerebral blood volume. Stroke. 1995;26(7):1234-1239.

4. Iida H, Itoh H, Nakazawa M, Hatazawa J, Nishimura H, Onishi Y, Uemura K. Quantitative mapping of regional cerebral blood flow using iodine-123-IMP and SPECT. J Nucl Med. 1994;35(12):2019-2030.

5. Mizumura S, Nakagawara J, Takahashi M, Kumita S, Cho K, Nakajo H, Toba M, et al. Three-dimensional display in staging hemodynamic brain ischemia for JET study: objective evaluation using SEE analysis and 3DSSP display. Ann Nucl Med. 2004;18(1):13-21.

6. Itoh S, Iwata A, Watanabe Y, Kitano S, Imai S, Yoshiya K. [Quantitative measurements of regional cerebral blood flow using technetium-99m-L,L-ECD SPECT activated with acetazolamide: fundamental study of measurement's accuracy, comparison with 123I-IMP ARG method]. Kaku Igaku. 1997;34(11):1047-1053.

7. Ogasawara K, Tomitsuka N, Kobayashi M, Komoribayashi N, Fukuda T, Saitoh H, Inoue T, et al. Stevens-Johnson syndrome associated with intravenous acetazolamide administration for evaluation of cerebrovascular reactivity. Case report. Neurol Med Chir (Tokyo). 2006;46(3):161-163.

8. Meguro T, Tanabe T, Muraoka K, Terada K, Hirotsune $\mathrm{N}$, Nishino S. [Enlargement of cerebral infarction after CBF study with acetazolamide challenge: two case report]. No Shinkei Geka. 2009;37(2):183-188.

9. Muroi C, Yonekawa Y, Khan N, Pangalu A, Keller E. Metabolic changes after $\mathrm{H}(2)$ 15O-positron emission tomography with acetazolamide in a patient with moyamoya disease: case report and review of previous cases. J Neurosurg Anesthesiol. 2003;15(2):131-139.

10. Shimotsu Y, Hayashida K, Hirose Y, Kume N, Nishimura T. Combined thallium-201 myocardial with technetium-99m-HMPAO brain SPECT: myocardial ischemia induced by acetazolamide in severe coronary artery disease. J Nucl Med. 1998;39(3):408-410.

11. Suzuki S, Ikeda S, Tamaru T, Onoue N, Tanaka M, Ishizuka T, Shinozaki T. A case of myocardial ischemia worsening during administration of acetazolamide. Kokyu to Junkan. 2010;58(10):1071-1074. (Japanese).

12. Shimotsu Y, Hayashida K, Kume N, Fukuchi K, Nishimura T. Acetazolamide induced myocardial ischemia in patients with severe coronary artery disease. Ann Nucl Med. 1998;12(1):21-27.

13. Vanninen E, Kuikka JT, Tenhunen-Eskelinen M, Vanninen R, Mussalo H. Haemodynamic effects of acetazolamide in patients with cardiovascular disorders: correlation with calculated cerebral perfusion reserve. Nucl Med Commun. 1996;17(4):325-330. 